\title{
Commentary on a new era of investigating 3D structure-based human-virus protein network dynamics
}

\author{
Shun Adachi ${ }^{1}$, Akio Adachi' ${ }^{2}$ and Masako Nomaguchi ${ }^{2 *}$ \\ 1 Multicellular Society Laboratory, Center for Frontier Research, National Institute of Genetics, Mishima, Japan \\ 2 Department of Microbiology, Institute of Health Biosciences, The University of Tokushima Graduate School, Tokushima, Japan \\ ${ }^{*}$ Correspondence: nomaguchi@basic.med.tokushima-u.ac.jp
}

\section{A commentary on}

Structural principles within the humanvirus protein-protein interaction network by Franzosa, E. A., and Xia, Y. (2011). Proc. Natl. Acad. Sci. U.S.A. 108, 10538-10543.

Virology is a fundamental research field for viruses that encompasses studies on minimum replication units, rapid evolution, and clinical applications. Similar to the other biological systems, structural study of viral system is based on three distinct phases of understanding: (I) modeling of steady-state network; (II) involvement of spatial confinements to the model; (III) involvement of timing asymmetry to the model. Most molecular biological understandings are limited to phase (I), rendering later phases to be investigated by future systems biology.

Even in phase (I), complexities of protein-protein network system make the researchers puzzled over examining actual underlying principles in the observed data sets. One type of solution for such an abstraction of the principles is to model a protein-protein interaction network based on graph theory; a theory of pair-wise relations between objects of a certain collection. Graph is constituted of nodes (for protein of interests in this case) and edges (for a certain protein-protein interaction), sometimes accompanied by different evaluation weights for an expected dynamics. Scalefree network, in which a small number of nodes act as hubs and the other nodes possess extremely small numbers of links, is supposed to be a characteristic of actual protein-protein interaction network in biological system. This type of network is prominent when the network encounters adding-on system for growth and selection bias for linking nodes act as hubs. However, it is not clear whether an input and output of such an abstracted system represents actual dynamics of original biological system, and what biological significances of parameters characterizing the networks are, i.e., density, transitivity, reciprocity, centrality etc. Progressing of the type of study requires further information to analyze the network elegantly.

In the article that deals with underlying principles of human-virus protein interaction network (Proc. Natl. Acad. Sci. U.S.A. 108, 10538-10543), Drs Franzosa and Xia have reported the results from $3 \mathrm{D}$ surface structure-based protein interaction network system. Based on 3039 endogenous interactions among 2435 human proteins as well as 53 exogenous interactions among 50 viral proteins from 36 viral species and their 50 human target proteins, they developed combined method of BLAST homology evaluation algorithm and MSMS program based on solvent-accessible surface area (SASA). The unique properties of this model case study are (i) detection of convergent evolution (mimicking evolution of viral protein surfaces to non-homological host human protein surfaces, in contrast to host gene duplication resulted in overlapping protein surface interactions) and evolutionary "arms race" (fast evolving trait of exogenous interactions due to cutting-off and catching-up interactions between human target proteins and viral proteins) due to rapid evolution and highly antagonistic manner of viral proteins and their target proteins, in contrast to cooperative interaction of endogenous human protein interactions; (ii) higher resolution of data based on more information from $3 \mathrm{D}$ surface structure interface. The original idea of using $3 \mathrm{D}$ surface information to network analysis is from the yeast study by Kim et al. (2006). In contrast to Kim's work, the patterns of evolution became much clearer based on fast evolving antagonistic viral proteins of absolutely different origins from host human. Compared to endogenous interactions, exogenous interactions exhibit transient structure, increase in number of interacting proteins, higher tendency of regulatory function, and faster evolution. Addition of BLAST information elucidates co-evolutionary information such as convergent evolution and evolutionary arms race.

Although the researchers are still struggling in phase (I), the application of 3D structural information to a graph of protein-protein network is very useful for investigating the evolutionary dynamics of the network of interests applying huge sum of mechanical interactive information to a simple graph of the interaction network. Additional investigation by commonly used BLAST algorithm will be useful expecting clinical application of drug design to highly conserved protein-protein interaction, instead of fast evolving highly adaptive region for target viruses.

\section{REFERENCE}

Kim, P. M., Lu, L. J., Xia, Y., and Gerstein, M. B. (2006) Relating three-dimensional structures to protein networks provides evolutionary insights. Science 314, 1938-1941

Received: 22 August 2011; accepted: 22 August 2011; published online: 08 September 2011

Citation: Adachi S, Adachi A and Nomaguchi M (2011) Commentary on a new era of investigating $3 D$ structurebased human-virus protein network dynamics. Front. Microbio. 2:186. doi: 10.3389/fmicb.2011.00186

This article was submitted to Frontiers in Virology, a specialty of Frontiers in Microbiology.

Copyright (c) 2011 Adachi, Adachi and Nomaguchi. This is an open-access article subject to a non-exclusive license between the authors and Frontiers Media SA, which permits use, distribution and reproduction in other forums, provided the original authors and source are credited and other Frontiers conditions are complied with. 